\title{
Primer registro de albinismo en la raya redonda enana, Urotrygon nana (Urotrygonidae: Elasmobranchii), en el Pacífico oriental
}

\author{
First record of albinism in the dwarf round stingray, Urotrygon \\ nana (Urotrygonidae: Elasmobranchii), in the eastern Pacific
}

Vicente Anislado-Tolentino ${ }^{1}$, Marcela G. Medina-Lerma ${ }^{2}$ y Luis Fernando Del Moral-Flores ${ }^{2 *}$

\section{RESUMEN}

El albinismo es una condición genética en la cual la proteína melánica está ausente o no es funcional y ocasionalmente ha sido reportado en el grupo de los condrictios. En este trabajo se describe el albinismo total en una raya redonda enana, Urotrygon nana, hembra madura de $81 \mathrm{~mm}$ del ancho de disco y con un peso de $18.42 \mathrm{~g}$, fue capturada por medio de arrastre de fondo a una profundidad de $40 \mathrm{~m}$ frente a Puerto Madero, Chiapas, Golfo de Tehuantepec, Pacífico oriental (1431' 6' N - 92 $\left.26^{\circ} 75^{\prime \prime} \mathrm{W}\right)$. Se cuantificaron las características morfométricas del ejemplar albino y se compararon con las de un espécimen normal del mismo sexo. Este trabajo representa el primer registro de esta anormalidad en la familia Urotrygonidae.

Palabras claves: Leucismo, Batoidei, Myliobatiformes, raya albina, anormalidad.

\begin{abstract}
Albinism is a genetic condition in which the melanin pigment protein is either absent or nonfunctional and has occasionally been reported in chondrichthyes. Total albinism in an 18.42 g female adult dwarf stingray, Urotrygon nana, with an $81 \mathrm{~mm}$ disc width is described in this paper. The subject was caught using bottom trawl methods $40 \mathrm{~m}$ deep off Puerto Madero, Chiapas, Gulf of Tehuantepec, eastern Pacific (14 31' 6' N - 92 $26^{\circ} 75^{\prime}$ ' W). The albino morphometric characteristics were compared with those of normal specimens of the same sex. This paper represents the first record of this abnormality in the family Urotrygonidae.
\end{abstract}

Keywords: Leucism, Batoidei, Myliobatiformes, albino stingray, abnormality.

\section{INTRODUCCIÓN}

El albinismo es una condición genética en la que la melanina, proteína de pigmento, está ausente o no es funcional (Reum et al. 2008). Es causado por una perturbación de la vía enzimática responsable de la producción de melanina (Ball et al. 2013). Se reconocen cuatro tipos de albinismo: (1) total o completo, en el

CECAT 03 Irapuato, Guanajuato. Dirección General de educación en Ciencia y Tecnología del Mar-SEP. Camino a Valencianita No. 637. Comunidad El Copal, CP. 36500 Irapuato. Guanajuato, México. anislado@gmail.com

2 Laboratorio de Zoología, Facultad de Estudios Superiores Iztacala, Universidad Nacional Autónoma de México (UNAM), Av. de los Barrios 1 Los Reyes Iztacala, Tlalnepantla, CP. 54090, Estado de México, México. margopolus@hotmail.com; delmoralfer@gmail.com*

Recibido: 31 de mayo de 2016

Corregido: 20 de agosto de 2016

Aceptado: 24 de agosto de 2016

DOI: http://dx.doi.org/10.15359/revmar.8-2.6 
cual falta melanina en todo el cuerpo, incluyendo sus estructuras anatómicas externas como piel, dentículos dérmicos e iris; (2) incompleto, el cual describe la ausencia de pigmento ya sea en la piel, dentículos o iris; (3) imperfecto, donde la pigmentación es reducida o diluida en las estructuras; y (4) parcial, que corresponde a la reducción o ausencia de coloración en zonas localizadas de la piel o algunas otras partes anatómicas (Clark, 2002; Berdeen \& Otis, 2011; Bigman et al. 2015). Estos últimos tres tipos de albinismo son también llamados leucismo (Clark, 2002). El albinismo ha sido reportado en el grupo de los condrictios, tanto en los tiburones como en las rayas y en una sola especie de quimera (Ben-Souissi et al. 2007; Reum et al. 2008; Bigman et al. 2015), aunque es un fenómeno menos frecuente en comparación con los peces óseos (Wakida-Kusunoki, 2015). En el Pacífico oriental ha sido documentado el albinismo en diferentes especies de condrictios (Herald, 1953; Herald et al. 1960; Cohen, 1973; Talent, 1973; Follett, 1976; Ebert, 1985; De JesúsRoldan, 1990; Sandoval-Castillo et al. 2006; Reum et al. 2008; Bejarano-Álvarez \& Galván-Magaña, 2013; Escobar-Sánchez et al. 2014; Bigman et al. 2015; Sancho-Vázquez et al. 2015), sin embargo, aún no se ha reportado para la familia Urotrygonidae. La familia está representada hasta el momento por 18 especies y se distribuyen en las aguas tropicales de ambas costas del continente americano (Eschmeyer \& Fong, 2016). Se caracterizan por tener un tamaño de pequeño a moderado (16-80 $\mathrm{cm}$ ), el ancho del disco no más que 1.3 veces el largo, aletas pectorales continuas y fusionadas, sin presencia de aletas dorsales, morro de redondeado a puntiagudo, habitan en mares tropicales y templados, pocas especies alcanzan profundidades mayores a los 100 m (Nelson et al. 2016). El objetivo de la presente contribución fue reportar el primer caso de albinismo para la familia Urotrygonidae, con un albinismo total para la especie Urotrygon nana Miyake \& McEachran 1988 recolectada en el Golfo de Tehuantepec, Pacífico oriental tropical.

\section{MATERIALES Y MÉTODOS}

El 18 de noviembre del 2004, durante las campañas de recolecta (FATE 0411-13) en la plataforma continental del Golfo de Tehuantepec, cerca de Puerto Madero, Chiapas (14' 31' 6' 'N - 92 $26^{\circ}$ 75 ' W), Pacífico oriental tropical, mediante el muestreo indirecto efectuado por barcos camaroneros a las 2:25 a.m. a una profundidad de $40 \mathrm{~m}$, se capturó un espécimen de $U$. nana con una coloración anormal. El organismo fue determinado a nivel específico con ayuda de las claves de Miyake \& McEachran (1988) y Castro-Aguirre \& Espinosa-Pérez (1996). Se obtuvieron los datos morfométricos del ejemplar anómalo y fueron comparados con un espécimen hembra sin albinismo, las medidas corresponden al protocolo de Montes-Domínguez (2001), estas son: longitud total (LT), que va del extremo anterior del hocico hasta el final de la aleta caudal; longitud del disco, de la punta del hocico al extremo posterior de la aleta pélvica; ancho 


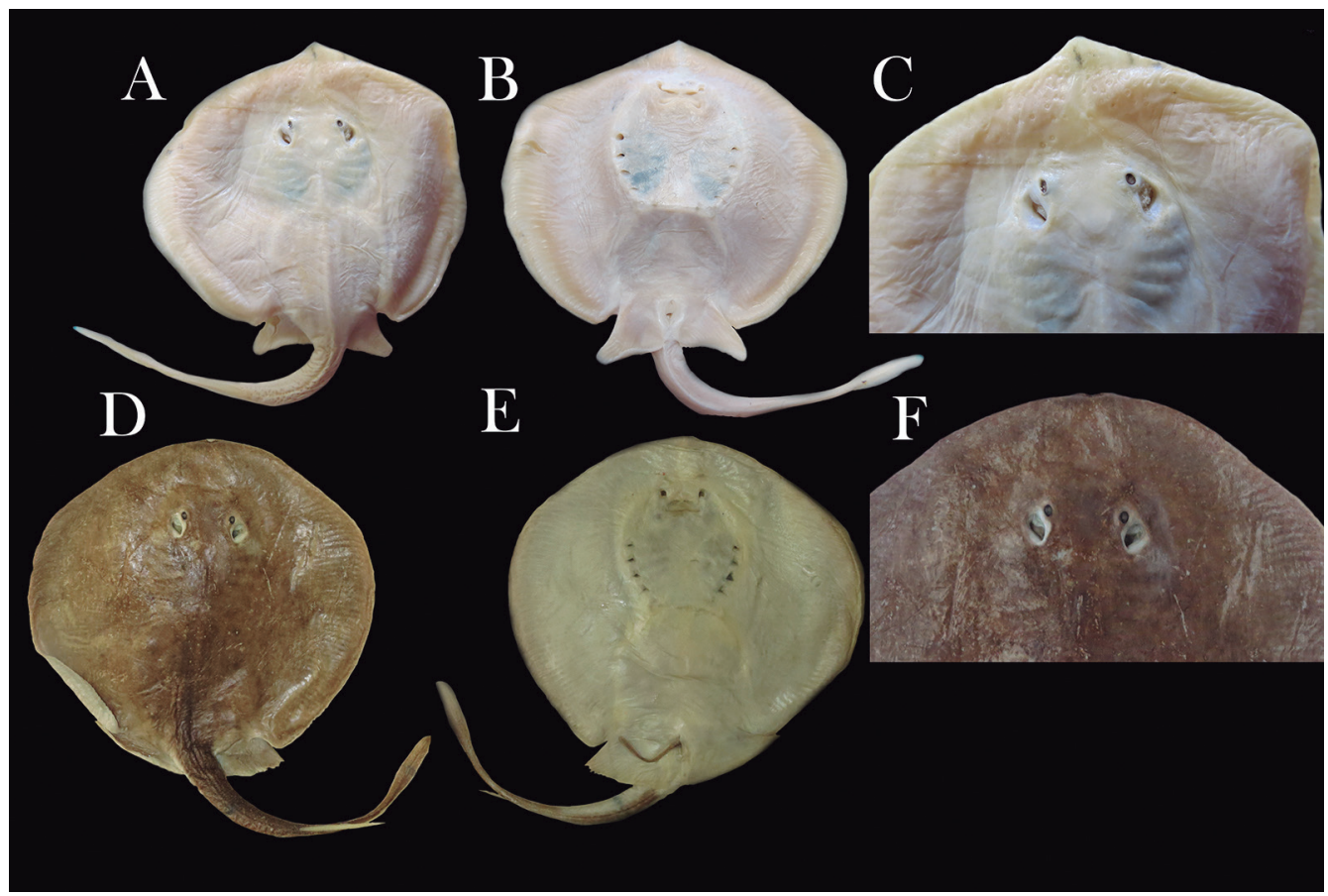

Fig. 1. Vista dorsal (A), ventral (B) y cefálica (C) de un ejemplar albino (hembra adulta, $139 \mathrm{~mm}$ de LT) y un ejemplar de pigmentación normal (hembra adulta, $245 \mathrm{~mm}$ de LT), vista dorsal (D), ventral (E) y cefálica (F) de Urotrygon nana

Fig. 1. Dorsal (A), ventral (B) and cephalic view (C) of an albino specimen (adult female $139 \mathrm{~mm}$ of TL) and a normally pigmented specimen (adult female, $245 \mathrm{~mm} \mathrm{TL}$ ), dorsal (D), ventral (E) and cephalic view (F) of Urotrygon nana

del disco, mayor distancia entre los márgenes distales de las aletas pectorales; diámetro del ojo, longitud máxima de los márgenes del ojo derecho; distancia interorbital, menor longitud entre las órbitas oculares; longitud preorbital del morro, de la punta del hocico al margen anterior del ojo; distancia interespiracular, menor longitud entre espiráculos; distancia del morro a la espina, de la punta del hocico al origen de la espina caudal; distancia de la boca a la cloaca, de la comisura mandibular al margen anterior de la cloaca; longitud del morro a la cloaca, de la punta del hocico al margen anterior de la cloaca; longitud del morro a la axila, del extremo anterior del hocico al final del margen posterior del disco; longitud caudal, del margen posterior del ano al final de la aleta caudal; longitud preoral del morro, de la punta del hocico a la comisura mandibular; distancia internarinal, longitud mínima entre narinas; distancia de la boca a cada hendidura branquial, de la comisura mandibular a la parte media de la respectiva hendidura branquial; distancia interbranquial, longitud mínima entre cada par de hendiduras branquiales; ancho de la boca, distancia máxima entre los pliegues bucales; 
Cuadro 1. Medidas morfométricas $(\mathrm{mm})$ del espécimen albino y uno de coloración normal de Urotrygon nana

Table 1. Morfometric measurements ( $\mathrm{mm}$ ) for the albino and normal pigmented specimens of Urotrygon nana

\section{Medidas (mm)}

\section{CIFI-1000 \\ (ejemplar albino)}

CIFI-1001

(ejemplar no albino)

Longitud total (LT)

Longitud del disco

139

73

81

1.36

11

20

15

99

44

61

65

70

16

8

10

13

17

20

21

22

21

18

15

14

7

110

18.42

Hembra

245

128

129

2.38

14

30

19

177

82

113

115

122

31

12

13

19

25

30

33

36

34

31

26

21

12

116

84.12

Hembra 
ángulo del hocico, grado de inclinación formado entre los márgenes anteriores del disco cefálico (Cuadro 1). El estado de madurez se determinó considerando los intervalos del ancho del disco propuesto por Nava-Nava (2013). Tanto el organismo de comparación (no. de catálogo: CIFI-1001) como el ejemplar albino (CIFI-1000) están en resguardo en la Colección Ictiológica de la Facultad de Estudios Superiores Iztacala, Universidad Nacional Autónoma de México, los cuales se encuentran preservados en alcohol etílico al 70\% (Fig. 1 D-F).

\section{RESULTADOS Y DISCUSIÓN}

En noviembre del 2004 se capturó un individuo hembra adulto de $U$. nana que presentó albinismo completo. El organismo tiene $139 \mathrm{~mm}$ de longitud total (LT), $81 \mathrm{~mm}$ de anchura total del disco y un peso de $18.42 \mathrm{~g}$ (Cuadro 1). Se corroboró la identidad a nivel específico por las siguientes características: ojos pequeños $(1.0 \%$ de la LT), cola delgada con espina, sin presencia de aletas dorsales, lóbulos caudales, alargados, ovales, superiores $(\sim 17 \%$ de LT) e inferiores no confluyentes, anchura máxima de la punta hocico de $48.60 \%$, ángulo del hocico entre $110^{\circ}$ y $130^{\circ}$, dentículos que van de 0.1 a $0.3 \mathrm{~mm}$ de altura en forma de cono o curvados (Miyake \& McEachran, 1988).

Son pocos los registros de albinismo en el orden Myliobatiformes (e.g., De Jesús-Roldan, 1990; Wakida-
Kusunoki, 2015), y ninguno en la familia Urotrygonidae, por lo tanto, el presente trabajo es el primer registro de presencia de albinismo en un espécimen de la familia y de $U$. nana. Sin embargo, se han reportado diferentes anormalidades dentro de esta familia como lo son: fusión y desarrollo completo de las aletas pectorales, organismos anoftalmos, neoplasia del hígado, ausencia de un mixopterigio, entre otras (Mejía-Falla et al. 2011; Torres-Huerta et al. 2016).

En la naturaleza han sido escasos los registros de albinismos en elasmobranquios, probablemente esta coloración los vuelve más vulnerables y presa fácil de depredadores (Talent, 1973). A diferencia del grupo de los tiburones, se ha reportado un mayor número de rayas en estado adulto con algún grado de albinismo (Ball et al. 2013; Wakida-Kusunoki, 2015), logrando alcanzar una mayor madurez al pasar desapercibidas al enterrarse en el sustrato marino en comparación con los organismos pelágicos (Ben-Souissi et al. 2007; Ball et al. 2013).

Aunque son aún pocos los registros de condrictios albinos se ha notado un incremento, debido posiblemente a un aumento natural de la población de organismos mutados (Walker \& Heessen, 1996; Dulvy et al. 2000; Ellis et al. 2005) o a la afectación por parte de contaminantes, como la presencia de organoclorados (Torres-Huerta et al. 2016). Las malformaciones pueden 
atribuirse a varias causas, siendo la contaminación del agua, por el aporte de agentes químicos, la más importante debido a las grandes descargas de aguas residuales de las zonas urbanas y las rurales (Haaker, 1977); además del aporte de afluentes cálidos de agua por parte de estaciones generadoras de electricidad, que incluso afecta la distribución de algunas especies de rayas (Hoisington \& Lowe, 2005). $\mathrm{Se}$ ha observado un mayor grado de afectación en las especies de rayas de hábitos costeros (De JesúsRoldan, 1990; Ben-Souissi et al. 2007; Wakida-Kusunoki, 2015) y al igual que otros organismos que viven enterrados en el sustrato como los peces planos (Gracian-Negrete \& Del Moral-Flores, 2013) presentan altas probabilidades de tener afectación en su descendencia al estar en contacto con altas concentraciones de metales pesados (Ochoa et al. 2016).

\section{AGRADECIMIENTOS}

Se agradece a L. A. Calderón Cortés (FES-I) por su ayuda para la obtención de las fotografías de los ejemplares de Urotrygon y a los revisores anónimos que ayudaron a enriquecer esta contribución.

\section{BIBLIOGRAFÍA}

Ball, R. E., Jones, C. S., Lynghammar, A., Noble, L. R. \& Griffiths, A. M. (2013). The first confirmed cases of full albinism in rajid species. J. Fish Biol., 82, 1433-1440. http://dx.doi.org/10.1111/ jfb. 12072
Bejarano-Álvarez, O. M. \& GalvánMagaña, F. (2013). First report of an embryonic dusky shark (Carcharhinus obscurus) with cyclopia and other abnormalities. Mar. Biodivers. Rec., 6(e11), 1-4. http://dx.doi.org/10.1017/ s1755267212001236

Ben-Souissi, J., Golani, D., Mérji, H., Ben-Salem, M. \& Capapé, C. (2007). First confirmed record of the Halave's Guitarfish, Rhinobatos halavi (Forsskål, 1775) (Chondrichthyes: Rhinobatidae) in the Mediterranean Sea with a description of a case of albinism in elasmobranch. Cah. Biol. Mar., 48, 67-75.

Berdeen, J. B. \& Otis, D. L. (2011). An observation of a partiallly albinistic Zenaida macroura (Mourning Dove). Southeast Nat., 10(1), 185-188. http:// dx.doi.org/10.1656/058.010.0117

Bigman, J. S., Knuckey, J. D. S. \& Ebert, D. A. (2015). Color aberrations in Chondrichthyan fishes: first record in the genus Bathyraja (Chondrichthyes: Rajiformes: Arhynchobatidae). Mar. Biodiv., 45, 1-9. http://dx.doi. org/10.1007/s12526-015-0403-Z

Castro-Aguirre, J. L. \& Espinosa-Pérez, H. (1996). Catálogo sistemático de las rayas y especies afines de México. Listados Faunísticos de México VII. México: Instituto de Biología, UNAM.

Clark, S. (2002). First report of albinism in the white-spotted bamboo shark, Chiloscyllium plagiosum (Orectolobiformes: Hemiscyllidae), with a review of reported color aberrations in elasmobranchs. Zoo. Biol., 21, 519-524. http://dx.doi.org/10.1002/zoo.10068

Cohen, J. L. (1973). An albino grey smoothhound Mustelus californicus, Gill. Calif. Fish Game, 59, 210-211. 
De Jesús-Roldan, M. (1990). An albino bat ray, Myliobatis californica, from the $\mathrm{Pa}-$ cific coast of Baja California Sur, Mexico. Calif. Fish Game, 76(2), 126-127.

Dulvy, N. K., Metcalfe, J. D., Glanville, J., Pawson, M. G. \& Reynolds, J. D. (2000). Fishery stability, local extinctions and shifts in community structure in skates. Conserv. Biol., 14, 283-293. http://dx.doi.org/10.1046/ j.1523-1739.2000.98540.x

Ebert, D. A. (1985). Color variation in the sevengill shark, Notorynchus maculatus Ayres along the California Coast. Calif. Fish Game, 71, 53-59.

Ellis, J. R., Dulvy, N. K., Jennings, S., Parker-Humphreys, M. \& Rogers, S. I. (2005). Assessing the status of demersal elasmobranchs in UK waters: a review. J. Mar. Biol. Assoc. U. K., 85, 1025-1047. http://dx.doi.org/10.1017/ S0025315405012099

Eschmeyer, W. N. \& Fong, J. D. (2016). Species by family/subfamily. Recuperado en mayo 21, 2016, disponible en http://researcharchive.calacademy. org/research/ichthyology/catalog/SpeciesByFamily.asp

Escobar-Sánchez, O., Moreno-Sánchez, X. G., Aguilar-Cruz, C. A. \& AbitiaCárdenas, L. A. (2014). First case of synophthalmia and albinism in the Pacific angel shark Squatina californica. J. Fish Biol., 85, 494-501. http:// dx.doi.org/10.1111/jfb.12412

Follett, W. I. (1976). First record of albinism in the leopard shark (Triakis semifasciata Girard). Calif. Fish Game, 62(2), 163-164.

Gracian-Negrete, J. M. \& Del MoralFlores, L. F. (2013). Anomalías en algunas especies del género Achirus (Pleuronectiformes: Achiridae). Rev. Zool., 24, 9-15.
Haaker, P. L. (1977). Abnormal vertebral development in northern anchovy, Engraulis mordax Girard. Calif. Fish Game, 63, 182-185.

Herald, E. S. (1953). The 1952 shark derbies at Elkhorn Slough, Monterey Bay, California. Calif. Fish Game, 39, 237-243.

Herald, E. S., Schneebeli, W., Green, N. \& Innes, K. (1960). Catch records for seventeen shark derbies held at Elkhorn Slough, Monterey Bay. Calif. Fish Game, 46, 59-67.

Hoisington, G. \& Lowe, C. G. (2005). Abundance and distribution of theround stingray, Urobatis halleri, near a heated effluent outfall. Mar. Environ. Res., 60, 437-453. http://dx.doi:10.1016/j. marenvres.2005.01.003

Mejía-Falla, P. A., Navia, A. F. \& Muñoz, L. A. (2011). First record of morphological abnormality in embryos of Urotrygon rogersi (Jordan \& Starks, 1895) (Myliobatiformes: Urotrygonidae) in the Tropical Eastern Pacific. Lat. Am. J. Aquat. Res., 39(1), 184-188.

Miyake, T. \& McEachran, J. D. (1988). Three new species of the stingray genus Urotrygon (Myliobatiformes: Urolophidae) from the eastern Pacific. Bull. Mar. Sci., 42(3), 366-375.

Montes-Domínguez, H. (2001). Sistemática de las especies del género Urotrygon (Myliobatoidei: Urolophidae). Tesis de Maestría en Ciencias, Universidad Nacional Autónoma de México.

Nava-Nava, P. (2013). Biología reproductiva de la raya enana, Urotrygon nana Miyake y McEachran, 1988, en las costas de Sinaloa, México. Tesis de Maestría en Ciencias, Universidad Nacional Autónoma de México.

Nelson, J. S., Grande, T. C. \& Wilson, M. V. H. (2016). 
Fishes of the World. Edmonton, Canada: Wiley \& Sons. http://dx.doi. org/10.1002/9781119174844

Ochoa-Díaz, M. R., Rodríguez-Romero, J., López-Martínez, J. \& Maldonado-García, M. C. (2016). First record of spine malformation of the round stingray Urobatis halleri off the Western Coast of Baja California Sur, Mexico. Mar. Biodivers. Rec., 9, 70. http:// dx.doi. 10.1186/s41200-016-0074-x

Reum, J. C. P., Paulsen, C. E., Pietsch, T. W. \& Parker-Stetter, S. L. (2008). First record of an albino chimaeriform fish, $\mathrm{Hy}$ drolagus colliei. Northwest. Nat., 89(1), 60-62. http://dx.doi.org/10.1898/10511733(2008)89[60:FROAAC]2.0.CO;2

Sancho-Vázquez, F., Del Moral-Flores, L. F. \& Mendoza-Vargas, O. U. (2015). Albinismo parcial en un embrión de tiburón puntas negras, Carcharhinus limbatus (Carcharhinidae: Chondrichthyes) del Pacífico oriental. Lat. Am. J. Aquat. Res., 43(1), 274-276. http://dx.doi.org/10.3856/ vol43-issue1-fulltext-25

Sandoval-Castillo, J., Mariano-Meléndez, E. \& Villavicencio-Garayzar, C.
(2006). New records of albinism in two elasmobranchs: the tiger shark Galeocerdo cuvier and the giant electric ray Narcine entemedor. Cybium, 30(2), 191-192.

Talent, L. G. (1973). Albinism in embryo gray smoothhound shark, Mustelus californicus, from Elkhorn Slough, Monterey Bay, California. Copeia, 1973, 595-597. http://dx.doi. org/10.2307/1443129

Torres-Huerta, A. M., Meraz, J., CarrascoBautista, P. E. \& Díaz-Carballido, P. L. (2016). Morphological abnormalities of round rays of the genus Urotrygon in the Gulf of Tehuantepec. Mar. Biodiv., 46(1), 309-315. http://dx.doi. org/10.1007/s12526-015-0347-3

Wakida-Kusunoki, A. T. (2015). First record of total albinism in southern stingray Dasyatis americana. Rev. Biol. Mar. Ocean., 50(1), 135-139. http:// dx.doi.org/10.1006/jmsc.1996.0135

Walker, P. A. \& Heessen, H. J. L. (1996). Long-term changes in ray populations in the North Sea. ICES J. Mar. Sci., 1085-1093. 